\title{
Efectos de la producción de semillas y la heterogeneidad vegetal sobre la supervivencia de semillas y el patrón espacio-temporal de establecimiento de plántulas en Araucaria araucana
}

\author{
Araucaria araucana temporal and spatial seedling establishment patterns: masting, seed \\ predation and understory vegetation effects
}

JAVIER SANGUINETTI ${ }^{1, *} \&$ THOMAS KITZBERGER ${ }^{2}$
${ }^{1}$ Parque Nacional Lanín, Administración de Parques Nacionales, E. Frei 749, 8370, San Martín de los Andes, Argentina ${ }^{2}$ INIBIOMA-CONICET y Laboratorio Ecotono, CRUB, Universidad Nacional del Comahue, Quintral 1250, 8400, Bariloche, Argentina
* Autor correspondiente: sanguinetti.javier@gmail.com

\begin{abstract}
RESUMEN
El establecimiento de plántulas puede estar limitado por la producción de semillas y asociada a la producción intermitente y sincronizada de semillas (masting) y/o por granivoría, o por falta de micrositios aptos para la germinación y crecimiento de las plantas. La dispersión y supervivencia de semillas desde el árbol madre pueden determinar el patrón espacial del establecimiento y la literatura propone varios modelos de distancia madre-plántula. En este trabajo se estudiaron los efectos del masting, de la depredación de semillas y de la vegetación sobre el establecimiento en A. araucana. A partir de datos sobre producción de conos, sobre supervivencia de semillas y mediante el registro de plántulas a escala del árbol en distintos micrositios se evaluó el patrón temporal y espacial del establecimiento. A. araucana presentaría un establecimiento en forma de pulsos, sincronizados entre árboles, y asociado al masting debido a un significativo aumento en la supervivencia de semillas. En micrositios densos, especialmente de bambúes, la regeneración se encuentra fuertemente inhibida por la excesiva depredación de semillas y no debido a competencia con los bambúes. El patrón distancia madre-plántula observado parecería ajustarse al modelo Janzen-Connell considerando el aumento de supervivencia de semillas con la distancia y el pico de establecimiento alejado del árbol madre. Sin embargo, se detectó una significativa diferencia en el patrón distancia madre-planta entre la regeneración joven y avanzada por lo cual habría luego del establecimiento otro patrón de mortalidad edad y distancia dependientes. Los resultados sugieren que la ocurrencia de regeneración en A. araucana se encontraría controlada por la producción de semillas mientras que la granivoría, y su interacción con la vegetación, modularían la intensidad del establecimiento.
\end{abstract}

Palabras clave: Araucaria araucana, establecimiento, masting, modelo de Janzen-Connell.

\begin{abstract}
Seedling establishment may be limited by a poor seed production and/or granivory, or the availability of microsites for germination and plant growth. Seed dispersal and survival from mother tree could determine the spatial pattern of seedling establishment and the literature proposes several distance mother-recruit models. We studied the effects of masting, seed predation and understory vegetation on seedling establishment in A. araucana. Using tree cone production and seed survival data and through seedling surveys in different microsites we evaluated the temporal and spatial establishment pattern. In the study site $A$. araucana showed a pulsed pattern for seedling establishment, synchronized among trees, and associated with masting due to an increase of seed survivorship. At dense microsites, mainly Chusquea bamboo, seedling regeneration was strongly inhibited by an excessive seed predation and not due to competition with the bamboo. The observed distance mother-recruit pattern mainly fits the Janzen-Connell model that considers the increase of seed survivorship with distance and the establishment peak occurrence at intermediate distances from the seeding tree. However, we detected a significantly difference on the distance mother-recruit between seedlings and saplings, presumably due to differential age- and distance- dependent mortality patterns. These results suggest that the occurrence of $A$. araucana regeneration is mainly controlled by seed production and granivory, and its interaction with vegetation, modulate the seedling establishment intensity.
\end{abstract}

Key words: Araucaria araucana, establishment, Janzen-Connell model, masting. 


\section{INTRODUCCIÓN}

Uno de los problemas fundamentales en ecología vegetal es determinar la importancia relativa que tienen la producción de semillas, su depredación, la disponibilidad de micrositios para la germinación y el establecimiento de plántulas en limitar el reclutamiento de individuos a la población (Grubb 1977, Crawley 1983). En particular, la dinámica de regeneración de coníferas adaptadas a la sombra frente a la competencia con angiospermas es de sumo interés en el contexto de las hipótesis sobre nichos de regeneración, sobre competencia-colonización y sobre dinámica de parches (Pickett \& Thompson 1978, Pickett \& White 1985, Bond 1989, Veblen, 1992, Tilman 1994). En este contexto, en la bibliografía se discuten las ventajas y desventajas adaptativas que presentan las semillas grandes frente a las pequeñas, tanto para gimnospermas como angiospermas (Coomes \& Grubb 2003, Moles \& Westoby 2004).

La semillazón (masting) es la producción sincronizada e intermitente de semillas en poblaciones de especies longevas (Janzen 1971, Kelly 1994). Los beneficios adaptativos obtenidos por las plantas y los efectos del masting sobre otros componentes del ecosistema están ligados a la extensión y sincronización espacial de este proceso (Curran \& Leighton 2000). Las causas y las ventajas selectivas del masting sincronizado han sido explicadas mediante diversas hipótesis en las últimas décadas (Janzen 1971, Waller 1979, Smith et al. 1990, Lalonde \& Roitberg 1992). Entre estas hipótesis se encuentran la relacionada con la Eficiencia de Polinización ("Pollination Efficiency") (Smith et al. 1990) y con la Saciedad de Depredadores ("Predator Satiation") (Janzen 1971, Silvertown 1980). Estas se basan en el principio de la Economía de Escala, donde concentrar el esfuerzo reproductivo en pocos eventos es más eficiente, por lo cual la evolución debería favorecer la ocurrencia de un ocasional e intenso evento reproductivo en lugar de varios pequeños y regulares (Kelly 1994).

Estudios en varias especies, que incluyeron depredación de semillas, sugieren que el fracaso en el reclutamiento en micrositios con vegetación densa se debe más a la presión de la granivoría que a la competencia interespecífica con dicha vegetación (Reader 1993, Edwards \&
Crawley 1999). La producción, dispersión y tamaño de la semilla pueden influir directamente sobre el patrón espacial de la "sombra de semillas" alrededor del árbol madre (Nathan \& Muller-Landau 2000, Nathan \& Casagrandi 2004). La existencia de sitios "seguros" para la supervivencia de semillas y para la germinación así como la actividad depredadora del ensamble de granívoros puede cumplir un rol clave en la regulación temporal y espacial del reclutamiento de plántulas, a partir de la modificación significativa del patrón de la "sombra de semillas" inicial (Clark et al. 1999, Silman et al. 2003). Este impacto puede limitar el reclutamiento de plántulas, en especial en especies que no sufren limitaciones ligadas al establecimiento de las mismas y a la herbivoría (Harper 1977).

En las últimas décadas se han propuesto diversos modelos sobre patrones de distancia madre-plántula denominados "Janzen-Connell”, "Hubell” y "McCanny" (Janzen 1970, Connell 1971, Hubbell 1980, McCanny 1985). Difieren en la importancia relativa que le asignan a la variación de la dispersión y de la predación afectada por la distancia al árbol madre y en la posibilidad o no de saciar a los granívoros cerca del mismo. El modelo Janzen-Connell postula el reclutamiento a distancias intermedias como consecuencia de una mayor tasa de depredación cerca del semillero por la atracción de los granívoros al sitio de mayor densidad de semillas. Por el contrario, si bien Hubbell (1980) coincide en que aumenta la supervivencia de semillas con la distancia al árbol madre, plantea que la mayor depredación cerca del semillero no compensa el incremento desproporcionado de semillas en el suelo producida por la dispersión a dichas distancias, con lo cual postula una reducción monotónica del reclutamiento con la distancia. Por su parte, McCanny (1985) propone la exacta compensación entre dispersión y depredación en los distintos intervalos de distancia, lo cual produciría una densidad constante de establecimiento alrededor del árbol madre o que la depredación de semillas decrece con la distancia, como consecuencia de la saciación de los granívoros cerca del árbol parental, en este último caso predice una disminución del establecimiento similar al propuesto por Hubbell.

Con este marco teórico y en base a los estudios sobre la dinámica de los bosques 
mixtos de Araucaria araucana (Molina) C. Koch y Nothofagus spp. y su relación con los disturbios (Veblen 1982, Burns 1993, Finckh \& Paulsch 1995, González et al. 2005), nos proponemos avanzar en el conocimiento de la estrategia de regeneración de A. araucana. Hasta el momento hemos observado un patrón sincronizado de masting (Sanguinetti \& Kitzberger 2008) y de depredación modulada por el masting y por la vegetación (Sanguinetti \& Kitzberger 2009); el objetivo actual es observar el efecto de dichos patrones sobre la abundancia y la distribución espacial del establecimiento alrededor del semillero. Las preguntas abordadas son a) ¿Existen pulsos de regeneración en $A$. araucana asociados al masting?; b) ¿Qué papel cumple sobre la regeneración en A. araucana la vegetación en el sotobosque, disminuye la disponibilidad de semillas o afecta el establecimiento de plántulas por competencia?; c) ¿A cuáles de los modelos distancia madre-plántula se asemeja más A. araucana?

\section{MÉTODOS}

\section{Especie y área de estudio}

Araucaria araucana (Molina) C. Koch es una conífera endémica, emergente y longeva (> 1200 años) de los bosques templados de América del Sur (Veblen 1982). Está adaptada a condiciones de estrés, es tolerante a la sombra, crece principalmente en suelos volcánicos o rocosos pobres en materia orgánica y presenta una serie de adaptaciones al fuego que le permiten mantener su distribución y abundancia relativa frente a especies más competitivas del género Nothofagus spp. (Veblen 1982, Burns 1993). Recientemente se determinó que A. araucana es una especies que realiza masting con una moderada fluctuación anual en la producción de conos por árbol (Coeficiente de variación poblacional $\left(\mathrm{CV}_{\mathrm{p}}\right)=0.95 \pm 0.05 ; \mathrm{n}=9$ años; rango $=2$ a 45 conos promedio por árbol), pero en forma altamente sincronizada entre árboles de una misma población y entre poblaciones a escala regional y al parecer inducido por condiciones de sequía dos años antes de la caída de semillas (Sanguinetti \& Kitzberger 2008). El masting en A. araucana parece estar vinculado evolutivamente con una mayor eficiencia de polinización dado que en estos años se produce mayor fecundación y número de semillas por cono respecto a años sin masting (135 \pm 8 (ES, $n$ = 31) semillas por cono, con un $99.4 \% \pm 0.4$ (ES) viables vs. $58 \pm 9(\mathrm{ES}, \mathrm{n}=22)$, con el 40.0 $\% \pm 6.6$ (ES)) (Sanguinetti 2008). La dispersión de las semillas es por gravedad y viento, y se limita a un radio de $13 \mathrm{~m}$ promedio alrededor del árbol madre. Las semillas comienzan a caer en marzo hasta junio y excepcionalmente en septiembre (Muñoz Ibáñez 1984, Sanguinetti 2008). El depredador de semillas predispersión principal es la cachaña o cotorra austral (Enicognathus ferrugineus) (Müller) que consume en promedio el 2 y $21 \%$ de las semillas del árbol en años con y sin masting, respectivamente (Shepherd et al. 2008). Sin embargo, los depredadores principales de las semillas de A. araucana lo constituyen un ensamble de al menos cuatro roedores que actúan en la etapa postdispersiva (Shepherd \& Ditgen 2005). Este ensamble está formado por el ratón topo grande (Chelemys macronyx) (Thomas), el ratón pelilargo (Abrotrix longipilis) (Waterhouse), el ratón colilargo (Oligoryzomis longicaudatus) (Bennett) y el ratón arborícola (Irenomys tarsalis) (Philippi) (Shepherd \& Ditgen 2005), quienes consumen globalmente entre el 30 y el $70 \%$ de las semillas que caen al piso y donde los años de masting provocan su saciedad, especialmente en sitios con menor cobertura vegetal (Sanguinetti \& Kitzberger 2009). De acuerdo con Shepherd \& Ditgen $\left(2008^{1}\right)$, quienes estudiaron la dispersión de semillas mediante cámaras automáticas e imanes colocados en ellas, A. longipilis podría cumplir un rol importante como dispersor de estas semillas dado que traslada, hacia micrositios favorables para la germinación y el establecimiento de plántulas, el $40 \%$ del total de las semillas colectadas por roedores. Algunas especies exóticas, como la rata de agua, la liebre europea, el conejo o el ciervo colorado, han invadido los bosques de Araucaria, principalmente preocupan el impacto potencial del jabalí (Sus scrofa) (Linnaeus) el cual invadió los bosques estudiados en la década de 1970

SHEPHERD JD \& RS DITGEN (2008) Piñones y mamíferos pequeños: predadores y dispersores en los bosques de Araucaria araucana. XXII Jornadas Argentinas de Mastozoología, noviembre 2008, Villa Giardino-Córdoba, Argentina. 
(Jaksic et al. 2002) y que consume hasta el $30 \%$ de las semillas caídas al suelo, especialmente en zonas con escasa vegetación en el sotobosque (Sanguinetti \& Kitzberger 2009).

El estudio fue realizado en el norte del Parque Nacional Lanín (Argentina) a los 39¹2'37” S y los 7109'15" O, a 1,100 msnm, en una zona de bosques mixtos de A. araucana - Nothofagus pumilio (Poepp. \& Endl) y A. araucana - Nothofagus antarctica (G.Forst). La precipitación es de aproximadamente $3,000 \mathrm{~mm}$ anuales. Los muestreos para el estudio se realizaron en 30 de las 250 hectáreas totales de bosque mixto existente. Este bosque constituye la continuación en Argentina de la masa forestal seleccionada por González et al. (2005) para los estudios sobre dinámica de fuego.

\section{Muestreo y análisis}

Efectos del masting sobre la abundancia y supervivencia de semillas en primavera

Por un lado se evaluó el efecto del masting, de la distancia al árbol y de la cobertura vegetal sobre la abundancia de semillas y su tasa de supervivencia al inicio del establecimiento de plántulas. Para ello en la primavera de 2006 y 2007, nueve meses después del comienzo de la caída de semillas, se estimó la densidad de semillas sobrevivientes y de brácteas al momento de la germinación. Los años 2006 y 2007 fueron de baja y alta productividad de conos, donde los árboles produjeron en promedio 12 y 35 conos, respectivamente (Sanguinetti \& Kitzberger 2008). Se marcaron 11 árboles hembra separados al menos 30 metros del semillero más cercano y por cada árbol se realizaron ocho transectas de 20 metros de largo y 1 metro de ancho dispuestas en forma radial. Se midió la abundancia de semillas sanas y de brácteas en parcelas de 1 $\mathrm{m}^{2}$ y se registró el tipo de micrositio en cada parcela. Los micrositios fueron clasificados según condiciones crecientes de cobertura en: 1) "Suelo Desnudo" (SD); 2) "Hojarasca" (HO); 3) plantas de hojas anchas y/o renovales de Araucaria (SA); 4) "Pastos Altos" (PA) y 5) "Arbustos" (AR) (caña o N. antarctica). Los micrositios posteriormente se dividieron en tres niveles de cobertura vegetal: Baja (SD y HO), Media (PA y SA) y Alta (AR). Como índice de supervivencia de semillas por parcela se utilizó la razón semillas:brácteas teniendo en cuenta la existencia de una correlación positiva y significativa entre el número de semillas y de brácteas en el cono $\left(\mathrm{F}_{(1,18)}=17.08 ; \mathrm{r}^{2}=0.487 ; \mathrm{r}\right.$ $=0.698 ; \mathrm{P}<0.001 ; \mathrm{y}=3.69 \mathrm{x}+82.7$; Sanguinetti 2008). A nivel del árbol, para la estimación de supervivencia de semillas por árbol, se utilizó la información disponible sobre el número de conos por árbol marcado y de estimación del número de semillas por cono para los años 2006 y 2007 (Sanguinetti \& Kitzberger 2008). Con estos datos se estimó el número total de semillas producidas por árbol marcado y la densidad promedio de semillas por $\mathrm{m}^{2}$ en un radio de 20 metros alrededor del árbol que potencialmente se hubiese dispersado sino hubiera habido depredación pre y postdispersión.

La abundancia de semillas sobreviviente en primavera no presentó una distribución normal y por lo tanto se utilizó el número de semillas por $\mathrm{m}^{2}$ como variable dependiente, la presencia o ausencia de masting y los micrositios agrupados por cobertura como factores y el número de conos por árbol y la distancia al árbol como covariables en un análisis con Modelos Lineales Generalizados (GLMs) asumiendo que los datos siguen una distribución de probabilidades de Poisson y que la relación no lineal entre la variable dependiente e independientes se ajusta a una función logarítmica.

Los posibles cambios en el patrón de supervivencia de semillas distancia dependiente entre años con distinta producción de conos, se evaluó a partir de una Prueba de Homogeneidad de Pendientes utilizando un Modelo Lineal General (GLM) considerando la razón semillas:brácteas por $\mathrm{m}^{2}$ como variable dependiente, al árbol, la ocurrencia de masting como factores y a la distancia como variable continua (covariable). La variable dependiente fue transformada con la aplicación del arco seno de la raíz cuadrada para cumplir con los supuestos de la normalidad en los datos (Sokal \& Rohlf 1994)

Existencia de pulsos de regeneración y nivel de sincronía de establecimiento entre árboles

Por otro lado, se evaluó la existencia de años con pulsos de reclutamiento de plántulas 
muestreando la regeneración hasta $2 \mathrm{~m}$ de altura. Utilizando transectas como las antes mencionadas en 16 árboles, se registró la distancia al árbol madre y la altura de 6,250 plántulas. El año de establecimiento de estas plántulas se estimó a partir de la extrapolación de la relación entre la altura y la edad, calculada previamente. Para ello se colectaron 3 individuos de cada intervalo de $2 \mathrm{~cm}$ entre los 0 y $20 \mathrm{~cm}$ de altura $(\mathrm{n}=30)$ y 3 individuos de cada intervalo de $10 \mathrm{~cm}$ entre los 20 y $200 \mathrm{~cm}$ de altura $(n=60)$. Estas muestras fueron obtenidas al azar y en diferentes árboles abarcando todas las situaciones ambientales existentes. La edad de las plantas fue estimada a partir del conteo de anillos siguiendo los procedimientos estándares (Daniels et al. 2007). Para determinar la proporción de renovales según su origen de regeneración (vegetativa o sexual) 110 individuos más fueron excavados, pero no quitados, y a todos se les registró su condición regenerativa. Con estos datos se estudió el grado de sincronización temporal en el establecimiento de plántulas entre árboles y el nivel de coincidencia entre la ocurrencia de regeneración y de la producción de semillas a partir de datos sobre producción de conos (periodo 2000-2007 para el rodal y 2004-2007 para los 11 árboles monitoreados; Sanguinetti \& Kitzberger 2008) y sobre recolección de semillas (1986-2007) (datos oficiales Dirección de Producción, Provincia del Neuquén y del Parque Nacional Lanín). Para evaluar estos aspectos se realizaron análisis de regresión lineal simple utilizando al número de plántulas establecidas por árbol y año $(\mathrm{N}=11)$ como variable dependiente y al número de conos por árbol por año como variable dependiente o el número de conos promedio por rodal por año para el análisis a escala del árbol y del rodal, respectivamente.

La existencia de pulsos de regeneración en los árboles monitoreados se analizó de dos maneras. Por un lado, se verificó la existencia de bimodalidad entre años en el número de plántulas establecidas por árbol entre 1986 y 2007. La confirmación de bimodalidad se realizó utilizando el método propuesto por Herrera et al. (1998). Para ello, el número de plántulas por árbol y por año se estandarizó a media igual 0 y desviación estándar igual 1 y a partir del agrupamiento de todos los árboles se construyó una distribución de frecuencias combinada. Si el establecimiento anual de plántulas de cada árbol fluctuara al azar alrededor de un valor específico entonces esperaríamos, por el teorema central del límite, que la distribución de frecuencias combinada no se aparte significativamente de una distribución normal con media 0. Para su comprobación, la misma fue sometida a una prueba de Kolmogorov Smirnov para verificar la ausencia de normalidad lo que confirmaría la existencia de bimodalidad (Herrera et al. 1998). No fue posible evaluar la existencia de multimodalidad en el establecimiento de plántulas debido a la limitada longitud de la serie temporal de los datos para utilizar métodos rigurosos como el procedimiento Bootstrap (Efron \& Tibshirani 1993).

Por otra parte, la confirmación de pulsos de regeneración se estudió a partir del análisis de sincronización temporal en el establecimiento de plántulas entre árboles a partir del análisis de correlación de Pearson entre todas las combinaciones de pares de árboles y la estimación de la proporción de pares significativamente correlacionados de manera positiva. Para la prueba de correlación se utilizaron todos los árboles muestreados y se ajustó el valor de significancia aplicando la prueba secuencial de Bonferroni (Sokal \& Rholf 1994).

Efecto de la vegetación en el sotobosque sobre el establecimiento de plántulas

Mediante el registro del tipo de micrositio donde creció cada plántula joven $(<20 \mathrm{~cm}$ de altura) muestreada (frecuencia observada) y el tipo de micrositio a cada metro radial dentro de las ocho transectas por árbol (frecuencia esperada), se analizó la existencia de un desvío de lo esperado en el establecimiento de plántulas según la disponibilidad de los tipos de cobertura vegetal. La desviación de la distribución de frecuencias de lo esperado según la abundancia relativa de cada cobertura vegetal se evaluó mediante la Prueba $G$ dado que se cuenta con 12 réplicas (árboles) (Sokal \& Rohlf 1994).

Por otra parte, se realizó un experimento sobre germinación de semillas excluyendo a los granívoros, para evaluar el efecto directo de la vegetación sobre el establecimiento de 
plántulas. Para ello, se colocaron 20 semillas dentro de clausuras triangulares de alambre de $20 \times 20 \times 20 \mathrm{~cm}$ que excluyeron a todos los vertebrados, estableciendo 3 réplicas por cada cobertura vegetal definida. Cada clausura fue colocada en árboles diferentes y luego de ocho meses se evaluó el porcentaje de germinación entre los niveles de cobertura. Se aplicó una Prueba Kruskal-Wallis utilizando al porcentaje de semillas germinadas por clausura como variable dependiente y a la cobertura vegetal como factor para verificar la existencia de diferencias significativas.

Patrón de distancia madre-recluta: importancia de la dispersión y la predación de semillas

Con el objeto de contrastar y verificar el ajuste de los datos empíricos del patrón de distancias madre-plántula en A. araucana con los modelos teóricos propuestos por Janzen-Connell, Hubbell y McCanny, se estimaron las curvas de dispersión y supervivencia de semillas y de establecimiento de plántulas jóvenes a partir del muestreo alrededor de 11 semilleros. La curva de dispersión de semillas se estimó a partir del conteo de brácteas por $\mathrm{m}^{2}$ sobre las ocho transectas radiales de $20 \mathrm{~m}$ dispuestas por árbol, dada la correlación entre brácteas y semillas por cono. Esta curva fue ajustada a una distribución exponencial negativa como lo sugieren diversos autores (Kot et al. 1996, Clark et al. 1998). La curva de supervivencia de semillas se estimó a partir de la tasa de supervivencia obtenida experimentalmente (Sanguinetti \& Kitzberger 2009), mientras que la curva de densidad de semillas sobrevivientes se obtuvo del muestreo realizado en primavera. Por último, la curva de establecimiento de plántulas se obtuvo a partir de los datos sobre abundancia de plántulas con alturas menores a $10 \mathrm{~cm}$ registradas en las ocho transectas radiales. Con el objeto de identificar cambios en las curvas de semillas sobrevivientes y de plántulas establecidas con respecto a las brácteas (semillas) dispersadas, se compararon las tres curvas y su grado de ajuste a la distribución exponencial negativa. Complementariamente se realizaron correlaciones de Spearman entre las tres variables para evaluar el nivel de concordancia entre las mismas. Todas las curvas fueron superpuestas gráficamente para discernir cuál de los modelos teóricos representa mejor a los datos siguiendo el marco conceptual propuesto por Nathan \& Casagrandi (2004). Se utilizaron los siguientes criterios para evaluar la congruencia de los datos con los tres modelos teóricos: a) la existencia o no de una moda en el establecimiento de plántulas alrededor del árbol madre; b) la distancia al árbol madre de la moda de establecimiento; c) la relación entre la supervivencia de semillas y la distancia al árbol madre. A su vez, se analizó, mediante una Prueba Kolmogorov-Smirnov, si el patrón madre-recluta difería según la edad de establecimiento comparando el patrón de la regeneración reciente (<30 años) con el de la regeneración avanzada ( $>30$ años).

\section{RESULTADOS}

Efectos del masting sobre la abundancia y supervivencia de semillas en primavera

Durante el año de masting 2007 la densidad de semillas presentes en la época de germinación fue 18 veces más alta que en el 2006 (media ( \pm ES) de 0.838 semillas $\mathrm{m}^{-2} \pm 0.046$ vs. 0.051 semillas $\mathrm{m}^{-2} \pm 0.006(\mathrm{n}=1760)$; Tabla 1$)$, mientras que la producción de semillas fue solo 3 veces mayor con una estimación promedio de 3.62 y 1.05 semillas por $\mathrm{m}^{2}$ si todas hubiesen sobrevivido a la depredación pre y postdispersión. La comparación entre la densidad de semillas observada y la máxima sin depredación indicaría que en el 2007 y 2006 sobrevivieron el $23.1 \%$ y el $4.9 \%$ de las semillas, respectivamente. La abundancia de semillas sobrevivientes varió significativamente según la cobertura vegetal y la distancia al árbol (Tabla 1). La densidad de semillas fue significativamente mayor en micrositios con baja o media cobertura vegetal respecto a los de alta cobertura (Fig. 1). La abundancia de semillas sobrevivientes se concentró en los primeros 5 metros del árbol, distancia a partir de la cual la misma decreció exponencialmente en micrositios con cobertura baja y media (Cobertura*Distancia, Tabla 1; ver más adelante Fig. 5). Se observó que, a nivel de árbol, cuanto mayor fue la producción de conos, la densidad de semillas sobrevivientes fue menor (Masting*Conos, Tabla 1) y se concentró aun más cerca del árbol 
madre (Distancia*Conos, Tabla 1). En 2006 estos efectos no fueron significativos.

Similares resultados fueron obtenidos para el índice de supervivencia de semillas (razón brácteas:semillas) (Tabla 2). El índice aumentó 7 veces durante el masting y varió hasta 5 veces entre árboles y solo en 2 de los 11 individuos evaluados no se observó un cambio significativo durante el 2007. El índice de supervivencia aumentó con la distancia a la fuente de semillas pero no se observó este efecto en todos los árboles por igual (Árbol*Distancia, Tabla 2). En particular durante el masting el efecto de la distancia se incrementó unas 4 veces aunque no para todos los árboles.

TABLA 1

Resultados del efecto del masting, de la cobertura vegetal, del número de conos por árbol (covariable) y de la distancia al árbol (covariable) sobre la abundancia de semillas en época de germinación evaluados mediante una Prueba de Homogeneidad de pendientes utilizando Modelo Lineal Generalizado (GLMs).

Effects of masting, cone number per tree, vegetation cover and distance to seeding tree on the abundance of seeds in spring evaluated by Slope-Homogeneity test using Generalized Lineal Models (GLMs).

\begin{tabular}{llcc}
\hline Efectos & gl & Wald c & P \\
\hline Masting & 1 & 1099.03 & $<0.0001$ \\
Cobertura & 2 & 245.62 & $<0.0001$ \\
Distancia & 1 & 34.43 & $<0.0001$ \\
Masting*Cobertura & 2 & 20.43 & $<0.0001$ \\
Masting*Distancia & 1 & 1.68 & 0.195 \\
Cobertura*Distancia & 2 & 12.28 & $<0.01$ \\
Masting*Cobertura*Distancia & 2 & 3.51 & 0.173 \\
Conos por árbol & 1 & 85.29 & $<0.0001$ \\
Masting*Conos & 1 & 9.51 & $<0.01$ \\
Distancia*Conos & 1 & 11.03 & $<0.001$ \\
Masting*Distancia*Conos & 1 & 3.56 & 0.059
\end{tabular}
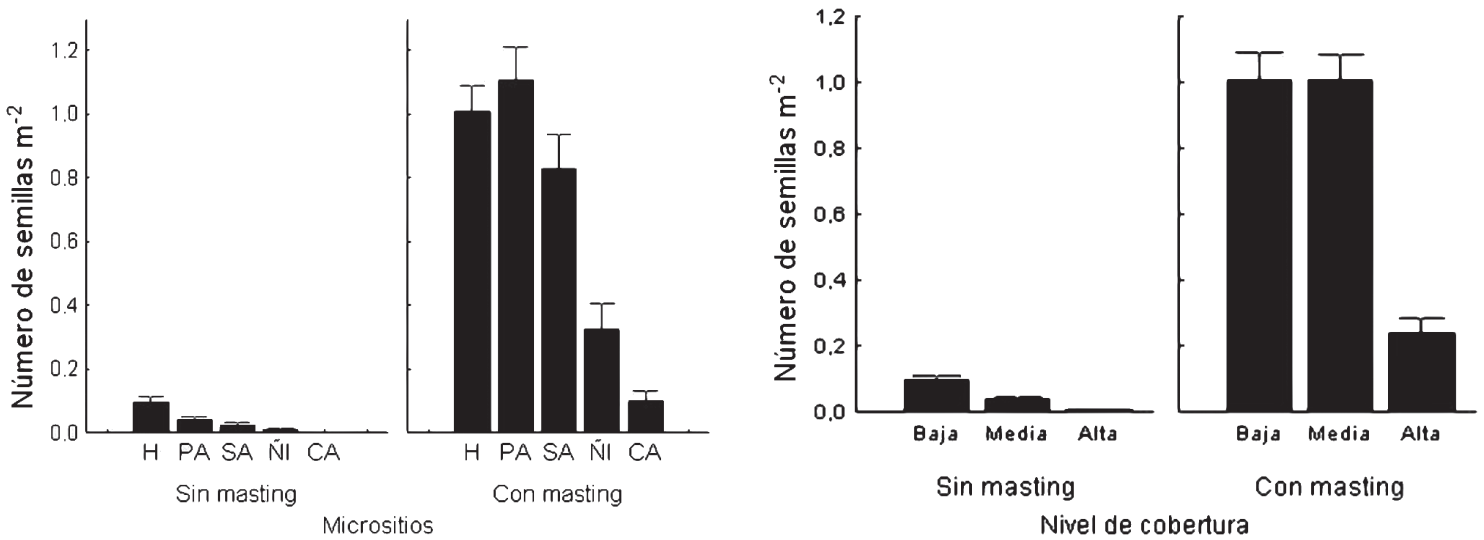

Fig. 1: Promedio ( \pm ES) de semillas por $\mathrm{m}^{2}$ sobreviviente a la época de germinación en 11 árboles monitoreados en 2006 y 2007: efectos del masting, del micrositio y de la cobertura vegetal. H: Hojarasca, PA: Pastos Altos, SA: Subarbustos, Ñi: Nire y CA: Caña.

Mean $( \pm$ SE) of seeds per sq m survived until germination period in 11 trees monitored between 2006 and 2007: masting, microsite and vegetation cover effects. H: Dead leaves, PA: High pasture, SA: Short shrubs, Ñi: Ñire shrubs, CA: Cane. 
TABLA 2

Efectos del masting, de la variabilidad entre árboles y de la distancia a la fuente de semillas sobre el cociente semillas:brácteas (tasa de supervivencia) examinados mediante una Prueba de Homogeneidad de pendiente en un Modelo Lineal General (GLM).

Effects of masting, variability among trees and distance to the seed source on the seed:scales ratio (survival rate) performed by a Slope-Homogeneity test using Generalized Lineal Models (GLMs).

\begin{tabular}{lccc}
\hline Efecto & $\mathrm{gl}$ & $\mathrm{F}$ & $\mathrm{P}$ \\
\hline Masting & 1 & 27.36 & $<0.0001$ \\
Árbol & 10 & 5.00 & $<0.0001$ \\
Distancia & 1 & 29.12 & $<0.0001$ \\
Masting*Árbol & 10 & 6.93 & $<0.0001$ \\
Masting*Distancia & 1 & 6.25 & 0.013 \\
Árbol*Distancia & 1 & 4.02 & $<0.0001$ \\
Masting*Árbol*Distancia & 10 & 4.17 & $<0.0001$ \\
\hline
\end{tabular}

Existencia de pulsos de regeneración y nivel de sincronía de establecimiento entre árboles

La relación entre la edad y la altura de las plántulas se ajustó significativamente a una regresión lineal $\left(\mathrm{F}_{(1,70)}=517.2 ; \mathrm{r}^{2}=0.881 ; \mathrm{P}<\right.$ $0.0001 ; \mathrm{y}=0.368 \mathrm{x}+1.086)$. El promedio de plántulas establecidas anualmente por árbol varió significativamente durante el período 1986-2007 $\left(\mathrm{F}_{(21,320)}=16.23 ; \mathrm{P}<0.0001 ;\right.$ Fig. 2$)$ pero en todos los años se observó regeneración, aunque hubo tres años donde en más del $50 \%$ de los árboles no se observó reclutamiento. La densidad promedio de plántulas establecidas en el año $0 \mathrm{y}+1$ de los tres eventos de masting del período fue significativamente más alta que en cualquier otro par de años de la serie temporal $(\mathrm{t}=4.82, \mathrm{gl}=387 ; \mathrm{P}<0.0001)$.

La frecuencia combinada de la distribución de plántulas establecidas por árbol para el conjunto de todos los árboles se apartó significativamente de la normalidad para una distribución con media 0 y desviación estándar igual a 1 (Prueba K.S .: D = 0.091; $\mathrm{P}<0.01$ ). La distribución de frecuencias combinada de las plántulas establecidas en todos los árboles en conjunto presentó dos modas distintas (Fig. 3). Una moda con valores estandarizados de establecimiento de plántulas relativamente bajos (entre -1.2 a -0.6 DS) y otra con valores altos (entre 1 y 1.7 DS) ambas sobrerrepresentadas en la muestra, mientras que los valores alrededor de la media esperada se encuentran subrepresentados.
El nivel de sincronización temporal entre árboles sobre el patrón de establecimiento de plántulas fue alto dado que más del $62 \%$ de los pares de árboles presentaron una correlación positiva significativa (Tabla 3 ). El coeficiente promedio de correlación de Pearson entre todos los árboles es de $0.69 \pm 0.02 \mathrm{ES}(\mathrm{n}=153)$. Esta proporción de pares de árboles con valores de correlación positiva significativa difiere de lo esperado por azar (Prueba Chi-cuadrado: $\chi^{2}=$ 186.39, $\mathrm{gl}=1 ; \mathrm{P}<0.001)$. El $38 \%$ de pares restantes con valores no significativos, presentaron una correlación promedio de $0.37 \pm$ 0.03 ES $(n=57)$ con solo cuatro pares con valores negativos.

La densidad de plántulas jóvenes establecidas por árbol en los 11 árboles monitoreados durante 4 años (período 2004-07) mostró una relación positiva tanto con respecto a la producción de conos en estos árboles $\left(\mathrm{r}^{2}=\right.$ $\left.0.101, \mathrm{r}=0.318, \mathrm{~F}_{(1,42)}=4.71 ; \mathrm{P}=0.03\right) \mathrm{como}$ con respecto al promedio de conos producidos entre todos los árboles del muestreo a escala del rodal $\left(\mathrm{r}^{2}=0.464, \mathrm{r}=0.681, \mathrm{~F}_{(1,42)}=36.38\right.$; $\mathrm{P}<0.0001$; Fig. 4). La regeneración de origen sexual y asexual difirió significativamente en términos de su proporción, de su patrón espacial y de su estructura de edades. El $10 \%$ de las plántulas registradas provinieron de rebrote de raíz; estas presentaron una altura (edad) significativamente mayor a las plántulas generadas por semilla (media $\pm(E S)$ de $97 \mathrm{~cm}$ \pm 14 vs. $\left.34 \mathrm{~cm} \pm 3 ; \chi_{(1)}{ }^{2}=16.34 ; \mathrm{P}<0.0001\right) \mathrm{y}$ se encontraron, en promedio $( \pm$ ES), 


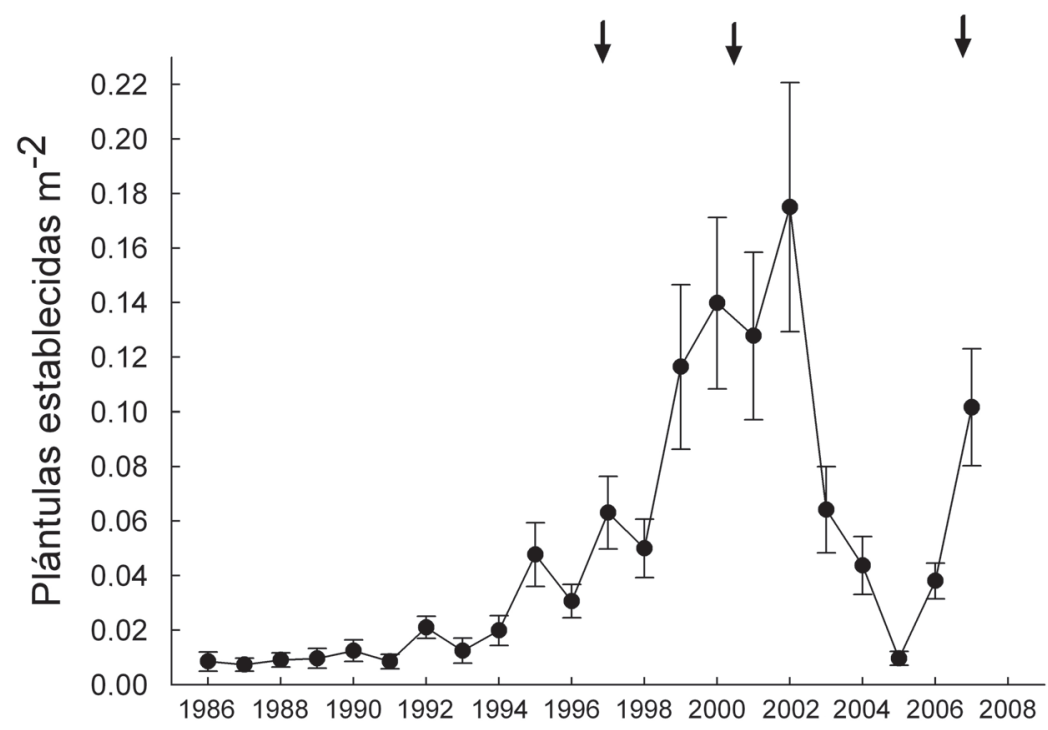

Año

Fig. 2: Variación anual en la densidad de plántulas de A. araucana establecidas entre 1986 y 2007 ( $\mathrm{N}=5814$ plántulas en 18 árboles). Las barras indican el error estándar de la media y las flechas muestras los años con masting.

Annual variation in A. araucana seedling density established between 1986 and 2007 ( $\mathrm{N}=5814$ seedlings in 18 trees). Horizontal bars show mean standard error and arrows show mast years.

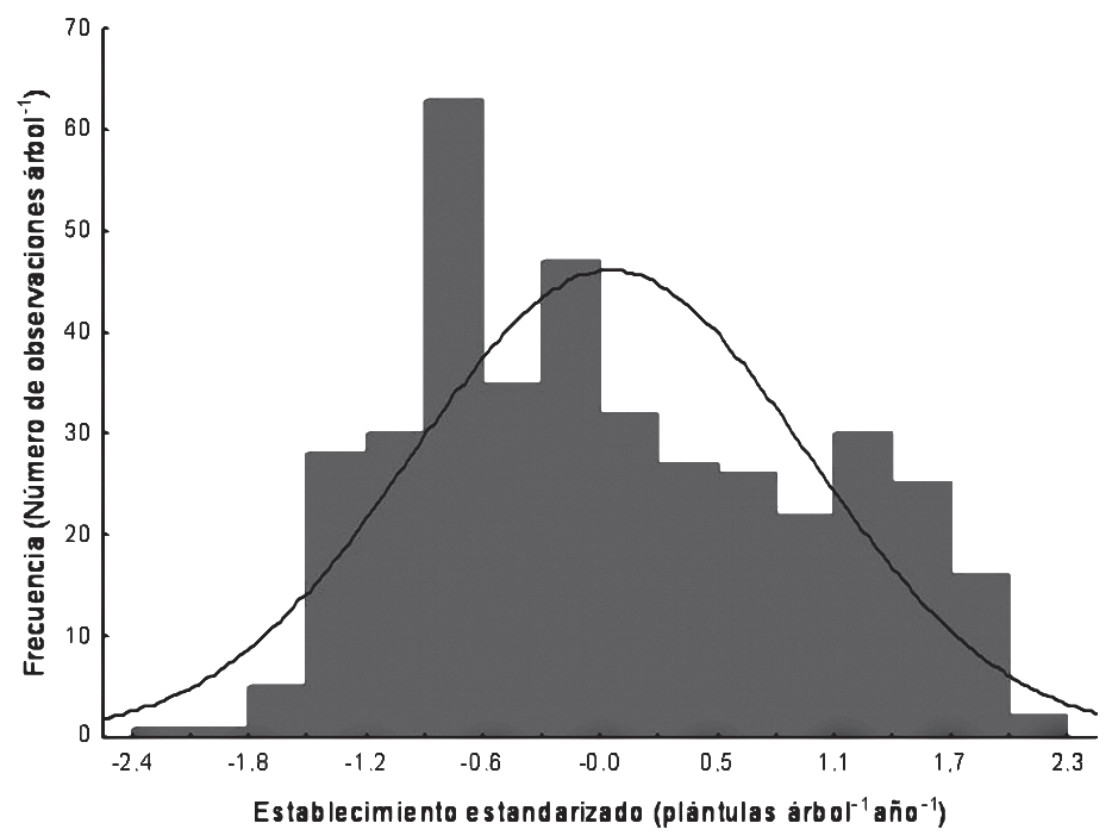

Fig. 3: Distribución de frecuencias estandarizadas (media $=0$ y $\mathrm{DS}=1$ ) del número de plántulas establecidas anualmente por árbol en el período 1986-2007 para el conjunto de árboles muestreados. Se muestra la curva normal esperada si el establecimiento por árbol tendiera a fluctuar alrededor de un valor promedio.

Standardized frequency distribution (mean $=0$ and SD $=1$ ) of annually established seedling numbers per tree for the19862007 period in all sampled trees. The normal distribution curve is shown expected if establishment fluctuates around a mean value. 


\section{TABLA 3}

Matriz de correlación entre árboles para el número de plántulas establecidas por año entre 19862007. Los valores en "negrita" indican coeficientes significativos $(\mathrm{P}<0.05)$ luego de aplicar el factor de corrección de Bonferroni.

Correlation matrix between trees for the number of seedlings established per year during 1986-2007. Values in bold represent significant coefficients $(\mathrm{P}<0.05)$ after Bonferroni correction.

\begin{tabular}{|c|c|c|c|c|c|c|c|c|c|c|c|c|c|c|c|c|c|}
\hline & $\mathrm{A} 2$ & A3 & A4 & A5 & A6 & A7 & A8 & A9 & A10 & A11 & A12 & A13 & A14 & A15 & A16 & A17 & A18 \\
\hline A1 & 0.94 & 0.85 & 0.50 & 0.50 & 0.71 & 0.63 & 0.84 & 0.89 & 0.86 & 0.75 & 0.83 & 0.68 & 0.81 & 0.25 & 0.56 & 0.98 & 0.98 \\
\hline A2 & & 0.93 & 0.41 & 0.57 & 0.58 & 0.60 & 0.84 & 0.80 & 0.79 & 0.63 & 0.73 & 0.62 & 0.66 & 0.43 & 0.47 & 0.93 & 0.92 \\
\hline A3 & & & 0.37 & 0.57 & 0.51 & 0.49 & 0.75 & 0.74 & 0.69 & 0.57 & 0.65 & 0.61 & 0.65 & 0.41 & 0.44 & 0.87 & 0.87 \\
\hline A4 & & & & 0.04 & 0.87 & 0.64 & 0.70 & 0.79 & 0.81 & 0.90 & 0.86 & 0.80 & 0.67 & -0.16 & 0.94 & 0.59 & 0.58 \\
\hline A5 & & & & & 0.16 & 0.26 & 0.31 & 0.36 & 0.30 & 0.15 & 0.31 & 0.30 & 0.18 & 0.33 & 0.06 & 0.44 & 0.43 \\
\hline A6 & & & & & & 0.73 & 0.81 & 0.92 & 0.90 & 0.98 & 0.95 & 0.89 & 0.85 & -0.21 & 0.95 & 0.75 & 0.78 \\
\hline A7 & & & & & & & 0.72 & 0.69 & 0.67 & 0.75 & 0.75 & 0.79 & 0.57 & 0.21 & 0.73 & 0.63 & 0.63 \\
\hline A 8 & & & & & & & & 0.88 & 0.93 & 0.85 & 0.88 & 0.84 & 0.69 & 0.13 & 0.74 & 0.88 & 0.87 \\
\hline A9 & & & & & & & & & 0.97 & 0.95 & 0.98 & 0.86 & 0.87 & 0.06 & 0.85 & 0.91 & 0.93 \\
\hline A 10 & & & & & & & & & & 0.93 & 0.97 & 0.84 & 0.79 & 0.07 & 0.93 & 0.89 & 0.89 \\
\hline A11 & & & & & & & & & & & 0.97 & 0.90 & 0.86 & -0.10 & 0.96 & 0.80 & 0.82 \\
\hline A 12 & & & & & & & & & & & & 0.89 & 0.83 & 0.00 & 0.89 & 0.87 & 0.87 \\
\hline A 13 & & & & & & & & & & & & & 0.68 & -0.04 & 0.89 & 0.71 & 0.74 \\
\hline A14 & & & & & & & & & & & & & & -0.08 & 0.75 & 0.84 & 0.87 \\
\hline A 15 & & & & & & & & & & & & & & & -0.19 & 0.25 & 0.20 \\
\hline A 16 & & & & & & & & & & & & & & & & 0.62 & 0.65 \\
\hline A17 & & & & & & & & & & & & & & & & & 0.99 \\
\hline
\end{tabular}

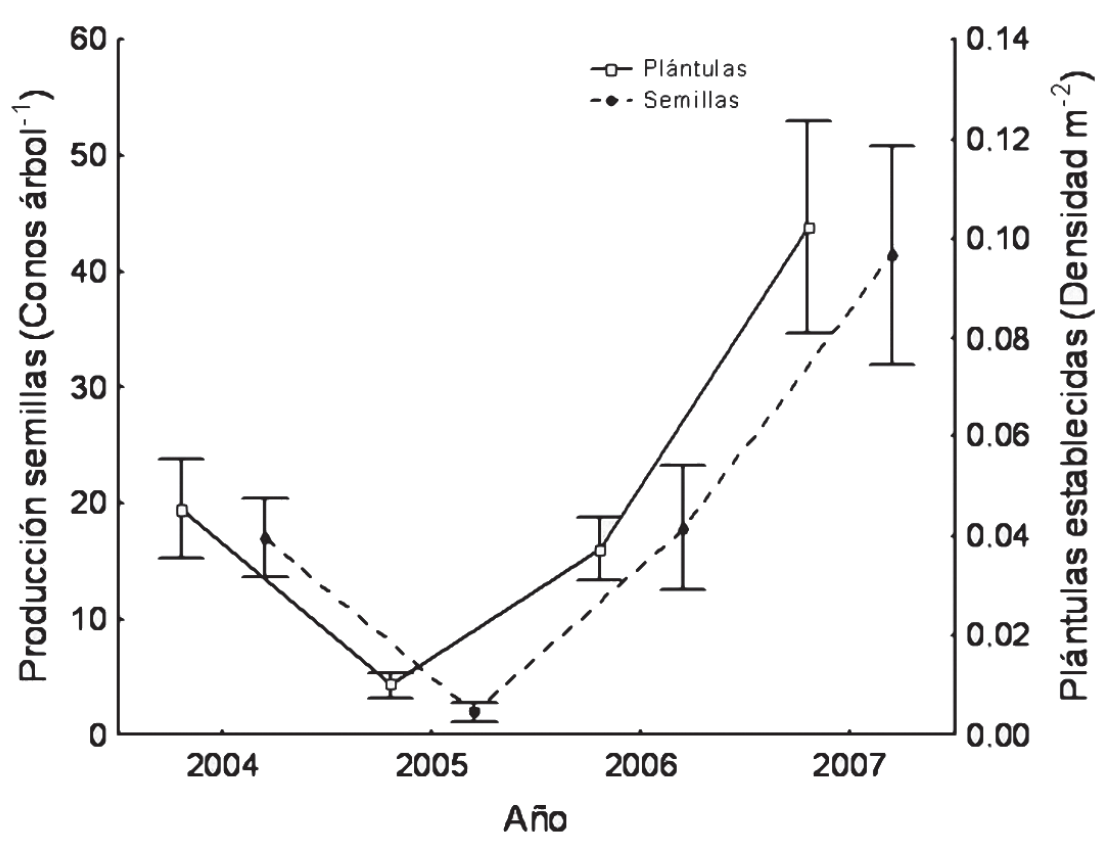

Fig. 4: Relación entre la densidad de plántulas por árbol $(\mathrm{N}=11)$ establecidas en el período 20042007 y la cantidad de conos producidos por árbol en el período.

Relationship between seedling density per tree $(\mathrm{N}=11)$ established within the 2004-2007 period and the abundance of cones produced per tree during this period. 
significativamente más cerca del árbol madre $\left(3.6 \mathrm{~m} \pm 1.1\right.$ vs. $7.3 \mathrm{~m} \pm 0.6 ; \chi_{(1)}^{2}=4.41 ; \mathrm{P}=$ $0.03)$. Esto último no difirió en los 6 árboles evaluados $\left(\chi_{(5)}{ }^{2}=6.95 ; \mathrm{P}=0.22\right)$.

\section{Efecto de la vegetación en el sotobosque sobre el establecimiento de plántulas}

Las frecuencias de establecimiento de plántulas jóvenes se apartaron significativamente de lo esperado según la cobertura vegetal disponible (Prueba G: $G_{p}$, Tabla 4). En sitios con alta y media cobertura vegetal se observó 4 veces menos y $40 \%$ más establecimiento del esperado, respectivamente. Sin embargo, estas respuestas no fueron homogéneas entre árboles $\left(\mathrm{G}_{\mathrm{H}}\right.$, Tabla 4). En micrositios con baja cobertura no se observó una tendencia clara, en dos árboles se observó un fuerte efecto negativo en el establecimiento pero en otros cinco la relación fue positiva (Tabla 4). Existió una relación lineal positiva entre el valor de $\chi^{2}$ obtenidos por árbol y la abundancia de plántulas jóvenes por árbol $\left(\mathrm{r}^{2}=0.681, \mathrm{r}=\right.$ $\left.0.825, \mathrm{~F}_{(1,10)}=21.38 ; \mathrm{P}<0.001\right)$.

El éxito de germinación de las semillas, al excluir los depredadores vertebrados, fue entre 60 y $95 \%$ y no varió significativamente entre los niveles de cobertura vegetal $\left(\mathrm{K}-\mathrm{W}_{(2,9)}=\right.$ 4.09, $\mathrm{P}=0.129)$. Similares resultados fueron observados en 2006 y 2007 en las semillas sobrevivientes monitoreadas en primavera donde más del $50 \%$, independientemente del micrositio, se encontraban germinadas.

\section{TABLA 4}

Efecto de la cobertura vegetal sobre el establecimiento de plántulas. Frecuencia observada (Fo) (micrositios con establecimiento) vs. Frecuencia esperada (Fe) (160 micrositios al azar por árbol). Prueba de Bondad de Ajuste $(\mathrm{G})$ a las frecuencias esperadas con todos los datos juntos $\left(\mathrm{G}_{\mathrm{P}}\right)$; Prueba de Heterogeneidad de frecuencias entre árboles $\left(\mathrm{G}_{\mathrm{H}}\right)$ y Prueba Global $\left(\mathrm{G}_{\mathrm{T}}\right)$. El signo de la desviación de lo esperado se muestra por árbol y cobertura/micrositio. SD: Suelo Desnudo, H: Hojarasca, PA: Pastos Altos, SA: Subarbustos/parche de renovales de araucaria, CA: Caña y Ni: Nirre.

Effect of vegetation cover on seedling establishment. Observed frequencies (Fo) (microsites with establishment) vs. Expected frequencies $(\mathrm{Fe})$ (160 random microsites per tree). Test for Goodness of fit $(\mathrm{G})$ to the expected frequency with the pooled data $\left(G_{P}\right)$; Test of Heterogeneity of frequencies between trees $\left(G_{H}\right)$ and Overall test $\left(G_{T}\right)$. Deviation sign from the expectation is shown per tree and vegetation cover/microsite. SD: Bare soil, H: Dead leaves, PA: High pasture, SA: Short shrubs or araucaria seedlings clumps, CA: Cane and Ñi: Nirre shrubs.

\begin{tabular}{|c|c|c|c|c|c|c|c|c|c|c|c|c|}
\hline \multirow[t]{2}{*}{ Árbol } & \multirow[t]{2}{*}{$\mathrm{G}\left(\chi^{2}\right)$} & \multirow[t]{2}{*}{$\mathrm{gl}$} & \multirow[t]{2}{*}{$\mathrm{P}$} & \multicolumn{3}{|c|}{ Baja $(\mathrm{SD}+\mathrm{H})$} & \multicolumn{3}{|c|}{ Media (PA+SA) } & \multicolumn{3}{|c|}{ Alta $(\mathrm{CA}+\tilde{\mathrm{N}} \mathrm{i})$} \\
\hline & & & & $\overline{\text { Fo }}$ & $\mathrm{Fe}$ & Signo & Fo & $\mathrm{Fe}$ & Signo & Fo & $\mathrm{Fe}$ & Signo \\
\hline 1 & 25.4 & 2 & $<0.001$ & 115 & 88 & + & 23 & 51 & - & 1 & 0 & \\
\hline 2 & 36.1 & 2 & $<0.001$ & 20 & 13 & + & 28 & 20 & + & 0 & 15 & \\
\hline 3 & 11.8 & 2 & $<0.01$ & 14 & 19 & & 56 & 45 & + & 1 & 7 & \\
\hline 4 & 2.4 & 2 & $>0.1$ & 3 & 3 & & 6 & 5 & & 0 & 1 & \\
\hline 5 & 68.1 & 2 & $<0.001$ & 39 & 17 & + & 33 & 14 & + & 29 & 69 & - \\
\hline 6 & 14.3 & 2 & $<0.01$ & 0 & 3 & & 19 & 10 & + & 25 & 31 & \\
\hline 7 & 48.4 & 2 & $<0.001$ & 65 & 38 & + & 24 & 23 & & 7 & 35 & - \\
\hline 8 & 32.4 & 2 & $<0.001$ & 60 & 42 & + & 107 & 113 & & 0 & 12 & \\
\hline 9 & 211.2 & 2 & $<0.001$ & 23 & 56 & - & 299 & 181 & + & 11 & 96 & - \\
\hline 10 & 94.7 & 2 & $<0.001$ & 37 & 106 & - & 184 & 116 & + & 6 & 4 & \\
\hline 11 & 130.6 & 2 & $<0.001$ & 22 & 29 & & 81 & 28 & + & 8 & 54 & - \\
\hline 12 & 6.5 & 2 & $<0.05$ & 49 & 55 & & 9 & 4 & + & 2 & 1 & \\
\hline $\mathrm{G}_{\mathrm{p}}$ & 168.5 & 2 & $<0.001$ & 447 & 471 & - & 869 & 610 & + & 90 & 325 & - \\
\hline $\mathrm{G}_{\mathrm{H}}$ & 659.6 & 22 & $<0.001$ & & & & & & & & & \\
\hline $\mathrm{G}_{\mathrm{T}}$ & 828.1 & 24 & $<0.001$ & & & & & & & & & \\
\hline
\end{tabular}


Patrón de distancia madre-recluta: importancia de la dispersión y la depredación de semillas

La densidad de brácteas (semillas) dispersadas decreció exponencialmente con la distancia al árbol madre y no difirió significativamente entre años $\left(\mathrm{y}_{(2006)}=74.6 \mathrm{e}^{-0.225 \mathrm{x}} ; \mathrm{F}_{(1,81)}=144.5\right.$; $\mathrm{P}<0.001 ; \mathrm{y}_{(2007)}=82.8 \mathrm{e}^{-0.212 \mathrm{x}} ; \mathrm{F}_{(1,81)}=158.9$; $\mathrm{P}<0.0001)$. La dispersión máxima promedio ( \pm ES) de brácteas estimada (D) no varió significativamente entre años a pesar de existir diferencias en la producción de conos $\left(\mathrm{D}_{2006}=\right.$ $13.4 \mathrm{~m} \pm 1$; rango: $9.1-18 \mathrm{~m}$ vs. $\mathrm{D}_{2007}=12.9$ $\mathrm{m} \pm 1$; rango $6.7-17.4 \mathrm{~m}$ ). Por su parte, tanto las semillas sobrevivientes a la primavera como las plántulas establecidas aumentaron en los primeros $3 \mathrm{~m}$ de radio alrededor de la fuente de semillas (Fig. 5). Se estimó una distancia máxima de dispersión de semillas promedio de 13 metros y un establecimiento de plántulas de corta distancia hasta $15 \mathrm{~m}$ del árbol. Los coeficientes de Spearman entre las distribuciones de brácteas, semillas y plántulas alrededor del árbol fueron significativas en todos los casos (brácteas-semillas: $\mathrm{r}_{\mathrm{s}}=0.745 ; \mathrm{P}$ $<0.001$; semillas-plántulas: $\mathrm{r}_{\mathrm{s}}=0.825 ; \mathrm{P}<$ 0.0001 ; brácteas-plántulas: $\mathrm{r}_{\mathrm{s}}=0.885 ; \mathrm{P}<$ $0.00001)$. Sin embargo, las distribuciones alrededor del árbol madre de semillas sobrevivientes y de plántulas jóvenes se alejaron de la distribución exponencial negativa observada en las brácteas (ln semillas vs. Distancia: $\mathrm{r}^{2}=0.242 ;$ ln plántulas vs. Distancia: $r^{2}=0.263$; ln brácteas vs. Distancia: $\left.r^{2}=0.679\right)$

La distribución espacial alrededor del árbol madre de la regeneración reciente $(<30$ años) y avanzada ( $>30$ años) difirió significativamente (Prueba de Kolmogorov Smirnov, $\mathrm{P}<0.001$ ) observándose en promedio $( \pm$ ES) menores distancias de establecimiento en las plantas más jóvenes (6.6 m \pm 0.07 vs. $8.5 \mathrm{~m} \pm 0.37$; Fig. 6 ).

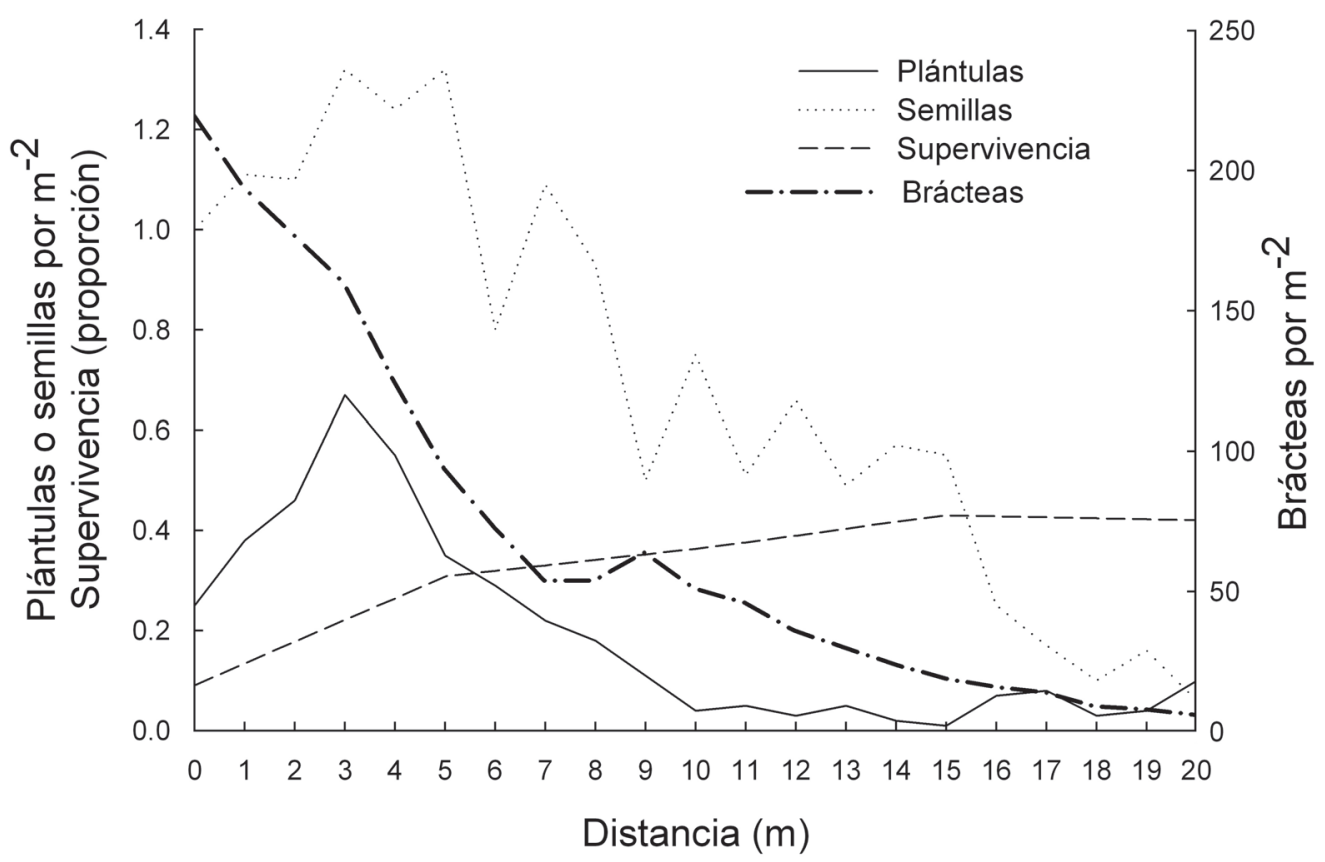

Fig. 5: Superposición de curvas sobre dispersión de brácteas, sobre tasa de supervivencia de semillas estimada a partir de (Sanguinetti \& Kitzberger, 2009), sobre abundancia de semillas en primavera y sobre establecimiento de plántulas jóvenes $(<10 \mathrm{~cm}$ de altura) para el masting del 2007. Las curvas representan el promedio de 11 árboles.

Superposed curves of scale dispersal, of seed survivorship estimated from data provided by (Sanguinetti \& Kitzberger, 2009), seed abundance in spring, and young $(<10 \mathrm{~cm}$ height) seedling establishment, estimated from 11 trees during the mast year 2007 . 

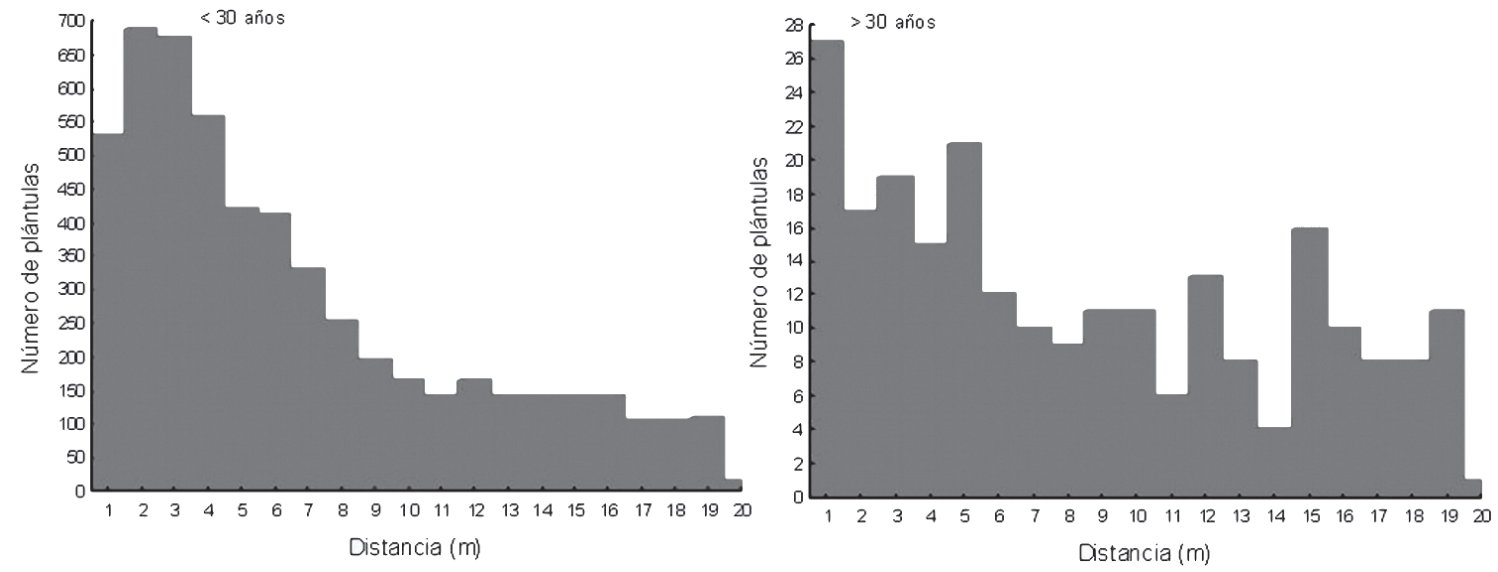

Fig. 6: Distribución de frecuencias de las distancias plántula-árbol madre para las plántulas establecidas entre 1971-2006 (< 30 años; $\mathrm{N}=5764$ ) y entre 1934-1970 (> 30 años; $\mathrm{N}=240)$.

Frequency distribution of seedling-tree distances for seedlings established between 1971 and $2006(<30$ years; N = 5764) and between 1934 and 1970 (> 30 years; $N=240$ ).

\section{DISCUSIÓN}

En el sitio de estudio y en el período de tiempo evaluado, el establecimiento de plántulas en $A$. araucana presentaría un patrón altamente sincronizado entre árboles y en forma de pulsos. La producción de conos sería la principal causa que explica la ocurrencia de este patrón de regeneración mientras que la depredación de semillas, y su interacción con la vegetación, modularían significativamente su intensidad. Los pulsos de establecimiento estarían asociados a los eventos de masting dado que el mismo provocaría un aumento significativo de la supervivencia de semillas. La distribución espacial de las plántulas alrededor del árbol madre se ajustaría al Modelo Janzen-Connell (Janzen 1970, Connell 1971), al presentar un patrón unimodal con el pico de establecimiento alejado de la fuente de semillas, producto del balance entre la dispersión y la depredación de semillas alrededor del semillero. Sin embargo, considerando que los resultados de este estudio provienen de un solo sitio y del análisis de un número limitado de árboles y que por ejemplo, la variable brácteas:semillas no sería del todo precisa como indicadora de supervivencia de semillas, dado que la regresión entre el número de brácteas y de semillas en el cono explica solo el $48 \%$ de la variación total, las conclusiones del presente trabajo no pueden generalizarse a todos los bosques de $A$. araucana y mayores investigaciones son necesarias para profundizar y precisar los actuales hallazgos.

\section{Producción de semillas y reclutamiento de plántulas}

El incremento significativo de semillas sobrevivientes y de la tasa de supervivencia de las mismas (razón brácteas:semillas) en años de masting, la detección de mayores niveles de establecimiento de plántulas en años próximos o inmediatamente posteriores a los eventos de masting en el período 1986-2007, la falta de normalidad en la distribución de frecuencias de establecimiento por árbol y el alto grado de sincronización en las fechas de establecimiento entre árboles, son fuertes evidencias que indicarían la existencia de pulsos sincronizados de establecimiento asociados al masting. Estos resultados concuerdan con el patrón de producción de conos altamente sincronizado entre árboles y poblaciones observados en esta conífera (Sanguinetti \& Kitzberger 2008). Similares resultados fueron obtenidos en diversas especies con semilla grande donde el masting favorece una mayor supervivencia de semillas y mayores probabilidades de establecimiento (Jensen 1982, Schupp 1990, Crawley \& Long 1995, Wolff 1996, Curran \& Leighton 2000, Turnbull et al. 2000).

La menor significancia entre el establecimiento de plántulas y la producción de 
conos observado a escala del árbol respecto a la escala del rodal podría responder a un patrón de supervivencia de semillas densidad dependiente (Sanguinetti \& Kitzberger 2009), donde los árboles con mayor productividad de conos sufren proporcionalmente mayores tasas de depredación de semillas que, según los resultados del presente trabajo, repercutirían en el establecimiento de plántulas. Sin embargo, la significativa relación encontrada en el análisis de regresión entre intensidad de establecimiento y número de conos utilizando los datos promedio a escala del rodal y la verificación de pulso de establecimiento luego del masting, sugeriría la existencia de escape de semillas a esta escala espacial. Por ende, a escala del árbol la interacción entre la oferta de semillas y el contexto local de la vegetación y de la depredación de semillas podrían ser la causa del éxito de establecimiento de plántulas mientras que a nivel poblacional tendría mayor incidencia la productividad global de semillas producidas cada año.

Supervivencia de semillas, heterogeneidad vegetal y establecimiento de plántulas

El efecto de los micrositios sobre la supervivencia de semillas frente a la granivoría y los resultados del experimento de germinación indicarían que la heterogeneidad vegetal modula indirectamente el establecimiento de plántulas en A. araucana en sitios con alta cobertura vegetal a través de la provisión de hábitat a los granívoros principales (roedores) y no a través de la competencia con la vegetación preexistente (competencia aparente). La alta significancia entre los valores de $\chi^{2}$ de la Prueba $G$ y la abundancia de plántulas por árbol indicaría que la segregación espacial de la regeneración provocada por la heterogeneidad vegetal, efectivamente estaría relacionada con procesos de mortalidad durante el estadío de semilla (e.g. depredación y/o falta de germinación) o durante la etapa temprana de desarrollo de la plántula. El establecimiento de plántulas particularmente en Chusquea culeou (Desvaux) se encuentra limitado por la intensa presión de depredación que ejercen los roedores (Sanguinetti \& Kitzberger 2009). Similares resultados se observaron en otros bosques de Quercus spp. con semilla grande y sotobosque de caña (Wada 1993). Los resultados de este trabajo muestran que $A$. araucana puede establecerse por semilla en cualquier tipo de micrositio si la granivoría es excluida o minimizada. En este sentido, se concuerda con Rechene et al. (2003) quien observó germinación y establecimiento de plántulas dentro de clausuras en la estepa en sitios desfavorables con suelo desnudo y plena irradiación solar. Por ende, el reclutamiento de plántulas en $A$. araucana estaría más limitado por la producción y predación de semillas que por factores abióticos reguladores del establecimiento. Esta situación se asemeja a lo observado en las especies del genero Quercus spp. donde el tamaño de semilla le otorga a las plántulas la independencia sobre la limitante de recursos (García \& Houle 2005).

Patrón de establecimiento de plántulas alrededor del árbol madre

En este trabajo se estimó una distancia promedio máxima de dispersión de semillas de 13 m que coincide con Muñoz-Ibáñez (1984) el cual la estimó a partir del distanciamiento de las plántulas. En árboles grandes con fustes mayores a $180 \mathrm{~cm}$ de diámetro y radio de copa de $10 \mathrm{~m}$, la dispersión de semillas y el establecimiento de plántulas pueden alcanzar los $17 \mathrm{~m}$. La corta distancia de dispersión máxima de semillas y la ausencia de una mejora de este parámetro durante los años de masting son claras evidencias de que $A$. araucana está fuertemente limitada en su capacidad de dispersión (Clark et al. 1998). Sin embargo, el efecto producido por el masting sobre la distribución espacial de semillas en comparación con la distribución de brácteas sugiere que en años con mayor productividad de conos aumenta la proporción de semillas sobrevivientes lejos del árbol.

La existencia de una relación lineal positiva y significativa entre la distribución de brácteas y plántulas demuestra la influencia de la dispersión de semillas sobre la distribución del establecimiento alrededor del árbol madre. Sin embargo, la pérdida de ajuste a la distribución exponencial negativa tanto en las semillas sobrevivientes como en las plántulas jóvenes establecidas indica que los procesos posteriores de mortalidad de semillas y plántulas son distancia dependientes (Clark et al. 1998). 
La disminución de la dispersión de semillas alrededor del árbol madre en forma exponencial, la supervivencia de semillas a mayores distancias del árbol madre y la existencia de un pico de establecimiento de plántulas a los 3-5 metros del semillero son importantes evidencias para postular que $A$. araucana se ajustaría al modelo JanzenConnell, al menos en zonas sin predominio de Chusquea culeou en el sotobosque. Al parecer, A. araucana no se ajustaría al modelo propuesto por Hubbell (1980) porque la dispersión de semillas no compensaría la mayor intensidad de depredación a cortas distancias del árbol madre y esto podría ser debido a la baja fecundidad de A. araucana donde en un año de masting el promedio de semillas por árbol es de apenas 6,500 semillas (Sanguinetti 2008). El modelo propuesto por McCanny (1985) es el menos plausible para $A$. araucana dado que la supervivencia de semillas ni decrece con la distancia ni el reclutamiento es constante a distintas distancias del semillero.

El cambio significativo en la distribución espacial alrededor del árbol madre de la regeneración avanzada podría deberse a la existencia de mortalidad de plántulas distancia y edad dependientes causada por otros factores que actuarían con posterioridad en el ciclo de vida e independientes de la granivoría. Estos factores serían la mortalidad de plántulas por estrés hídrico y/o lumínico contra los cuales la especie presenta importantes adaptaciones (Bekessy et al. 2002, Burgman et al. 2002, Lusk et al. 2006), la mortalidad por daño mecánico producido por la caída masiva de ramas desde el semillero, especialmente en inviernos con fuertes nevadas donde se midió un rango de 4 a 10 ramas completas caídas por árbol (Sanguinetti 2008) y la mayor incidencia de fuegos superficiales de baja intensidad y alta frecuencia (González et al. 2005) cerca del árbol madre por la acumulación de este combustible que podrían modificar el patrón de distribución espacial inicial provocado por la dispersión y la granivoría. El presente estudio se llevó a cabo en la porción argentina del bosque estudiado por González et al. (2005) en Chile, en el cual encontramos que el $39 \%$ de las plántulas relevadas de más de $20 \mathrm{~cm}$ de altura presentaron al menos 1 reiteración vegetativa desde el cuello del tallo mientras que el $26 \%$ presentaron 2 o más reiteraciones, lo cual constituye una evidencia de la ocurrencia de este tipo de estrés hídrico, lumínico, mecánico y/o por fuegos de superficie.

En síntesis, el patrón del masting en $A$. araucana al parecer inducido por el clima $\mathrm{y}$ asociado a una mayor eficiencia de polinización y con la saciedad de los granívoros (Sanguinetti \& Kitzberger 2008, Sanguinetti 2008, Sanguinetti \& Kitzberger 2009) produciría pulsos de establecimiento de plántulas igualmente sincronizados. A araucana tendría un umbral mínimo de producción de conos a nivel poblacional para compensar las perdidas por depredación pre y postdispersión de semillas producidas por Enicognathus ferrugineus y por el ensamble de granívoros y otros mamíferos introducidos por debajo del cual prácticamente no ocurre establecimiento de plántulas. Por encima de este umbral, que estaría en menos de 6 conos promedio por árbol, existiría un rango en la productividad de conos donde la depredación de semillas regularía la magnitud del establecimiento de plántulas, y en donde a baja densidad de semillas decrece el establecimiento al no existir posibilidades de compensación de la mortalidad producida por los factores posteriores que actúan sobre las plántulas. En años de masting, A. araucana lograría la saciedad de los granívoros y en el verano siguiente el establecimiento de plántulas sería máximo probablemente al existir procesos densidad dependientes que actúan sobre el desempeño de las plántulas y que permitirían compensar la mortalidad en las etapas posteriores al estadío de semilla.

A las adaptaciones de los adultos de $A$. araucana frente a los disturbios alógenos y su capacidad para persistir en el sitio y competir con Nothofagus spp., y a la mayor resistencia a la sombra de la regeneración avanzada como estratega r (Veblen 1982, Burns 1991) deberían sumársele el tamaño de la semilla y su patrón de producción de conos. El tamaño de semilla en $A$. araucana sería de vital importancia para la supervivencia de plántulas en sus etapas tempranas de desarrollo en sitios habitualmente con estrés ambiental (Lusk et al. 2006) mientras que su patrón de producción de conos garantiza una mayor polinización y supervivencia de semillas a pesar de su tamaño. 
De esta forma y considerando la longevidad de la especie, A. araucana acumula diferentes cohortes de plántulas debajo del semillero, mayormente en sitios con baja cobertura vegetal, que tienden a sobrevivir más hacia el límite de la sombra de semilla, donde dependen de la apertura de pequeños claros para finalizar el desarrollo de la regeneración avanzada y posteriormente alcanzar el dosel según la teoría sobre dinámica de parches (Burns 1991).

La desventaja en la menor producción de semillas en $A$. araucana al existir una mayor presión de selección para mantener un determinado tamaño de semilla podría ser suficientemente compensado por la longevidad y por el nivel de ocupación que muestra la especie en sus distintas poblaciones, evitando que Nothofagus spp. pueda desarrollar todo su potencial de aporte en densidad de semillas por unidad de superficie y por ocupación de cobertura en el dosel (Moles \& Westoby 2004). A su vez, los beneficios de una semilla grande en $A$. araucana parecería claramente transferirse a la plántula por varios años, no solo en el primer año de desarrollo, aspecto que también es fundamental considerar cuando se evalúa el balance entre tamaño y número de semillas en una especie determinada (Coomes \& Grubb 2003). La coexistencia de $A$. araucana y Nothofagus spp. se basa en la clara diferenciación de las historias de vida centrada en la capacidad de los adultos de sobrevivir y en las características de sus semillas y de sus plántulas y en la partición de los recursos entre especies.

\section{AGRADECIMIENTOS}

Los autores agradecen a Santiago Quiroga y a los voluntarios de Global Vision International (GVI) por su ayuda durante el trabajo de campo. Este trabajo es parte de la Tesis Doctoral de JS y fue financiado por el programa Russell Train Scholarship del Fondo Mundial para la Naturaleza (WWF), por el gobierno alemán (Ministerio GMZ), por el Instituto Internacional sobre Recursos Genéticos en Plantas (IPGRI), por la Agencia de Cooperación Internacional Española (AECI, proyecto CGL2004-0176-Feder coordinado por el Dr. Salvador J. Peris, Universidad de Salamanca) y por el Parque Nacional Lanín.

\section{LITERATURA CITADA}

BOND WJ (1989) The tortoise and the hare: ecology of angiosperm dominance and gymnosperm persistence. Biological Journal of the Linnean Society 36: 227-249.

BEKESSY SA, D SLEEP, A STOTT, M MENUCCINI, P THOMAS, et al. (2002) Adaptation of monkey puzzle to arid environments reflected by regional differences in stable carbon isotope ratio and allocation to root biomass. Forest Genetics 9: 63-70.

BURNS BR (1993) Fire-induced dynamics on Araucaria araucana - Nothofagus antarctica forest in the southern Andes. Journal of Biogeography 20: 669685.

CLARK JS, E MACKLIN \& L WOOD (1998) Stages and spatial scales of recruitment limitation in southern Appalachian forests. Ecological Monographs 68: 213-235.

CLARK JS, M SILMAN, R KERN, E MACKLIN \& J HILLERSLAMBERS (1999) Seed dispersal near and far: patterns across temperate and tropical forests. Ecology 80: 1475-1494.

COOMES DA \& PJ GRUBB (2003) Colonization, tolerance, competition and seed-size variation within functional groups. Trends in Ecology and Evolution 18: 283-291.

CONNELL JH (1971) On the role of natural enemies in preventing competitive exclusion in some marine animals and in rain forests. En: den Boer PJ \& GR Gradwell (eds) Dynamics of population: 298-312. Centre for Agriculture Publishing and Documentation, Wageningen.

CRAWLEY MJ (1983) Herbivory: the dynamics of animal-plant interaction. Blackwell Scientific Publications, Oxford, UK. 437 pp.

CRAWLEY MJ \& CR LONG (1995) Alternate bearing, predator satiation and seedling recruitment in Quercus robur L. Journal of Ecology 83: 683-696.

CURRAN LM \& M LEIGHTON (2000) Vertebrate response to spatiotemporal variation in seed production of mast-fruiting Dipterocarpaceae. Ecological Monographs 70: 101-128.

DANIELS LD, TT VEBLEN \& R VILLALBA (2007) Use of thin sections to improve age estimates of Nothofagus pumilio seedlings. Ecoscience 14: 1722.

EDWARDS GR \& MJ CRAWLEY (1999) Rodent seed predation and seedling recruitment in mesic grassland. Oecologia 118: 288-296.

EFRON B \& RJ TIBSHIRANI (1993) An introduction to the bootstrap. Chapman \& Hall, New York. 436 pp.

FINCKH MA \& A PAULSCH (1995) The ecological strategy of Araucaria araucana. Flora 190: 365382.

GARCIA D \& G HOULE (2005) Fine-scale spatial patterns of recruitment in red oak (Quercus rubra): What matters most, abiotic or biotic factors? Ecoscience 12: 223-235.

GONZÁLEZ ME, TT VEBLEN \& JS SIBOLD (2005) Fire history of Araucaria - Nothofagus forests in Villarica National Park, Chile. Journal of Biogeography 32: 1187-1202.

GRUBB PJ (1977) The maintenance of species richness in plant communities: the importance of the regeneration niche. Biological Review 52: 107-145.

HARPER JL (1977) Population biology of plants Academic Press, London, U.K. 892 pp.

HERRERA CM, P JORDANO, J GUITIAN \& A TRAVESET (1998) Annual variability in seed 
production by woody plants and the mast concept: Reassessment of principles and relationship to pollination and seed dispersal. American Naturalist 152: $576-594$

HUBBELL SP (1980) Seed predation and the coexistence of tree species in tropical forests. Oikos 35: 214229.

JAKSIC FM, JA IRIARTE, JE JIMÉNEZ \& DR MARTÍNEZ (2002) Invaders without frontiers: cross-border invasions of exotics mammals. Biological Invasions 4: 157-173.

JANZEN DH (1970) Hervibores and the number of tree species in tropical forests. American Naturalist 104 501-528.

JANZEN DH (1971) Seed predation by animals. Annual Review of Ecology and Systematics 2: 465-492.

JENSEN TS (1982) Seed production and outbreaks of noncycling rodent populations in deciduous forests. Oecologia 54: 184-192.

KELLY D (1994) The evolutionary ecology of mast seeding. Trends in Ecology and Evolution 9: 465470.

KOT M, MA LEWIS \& P VAN DEN DRIESSCHE (1996) Dispersal data and the spread of invading organisms. Ecology 77: 2027-2042.

LALONDE RG \& BD ROITBERG (1992) On the evolution of masting behavior in trees: predation or weather? American Naturalist 139: 1293-1304.

LUSK H, DS FALSTER, M PÉREZ-MILLAQUEO \& A SALDAÑA (2006) Ontogenetic variation in light interception, self-shading and biomasa distribution of seedlings of the conifer Araucaria araucana. Revista Chilena de Historia Natural 79: 321-328.

MCCANNY SJ (1985) Alternatives in parent-offspring relationships in plants. Oikos 45: 148-149.

MOLES AT \& M WESTOBY (2004) Seedling survival and seed size: a synthesis of the literature. Journal of Ecology 92: 372-383.

MUÑOZ-IBÁÑEZ R (1984) Análisis de la productividad de semillas de Araucaria araucana (Mol.) C. Koch, en el Área de Lonquimay - IX Región. Tesis de grado, Facultad de Ciencias Agrarias, Veterinarias y Forestales, Escuela de Ciencias Forestales, Universidad de Chile. 98 pp.

NATHAN R \& HC MULLER-LANDAU (2000) Spatial patterns of seed dispersal, their determinants and consequences for recruitment. Tree 15: 278-285.

NATHAN R \& R CASAGRANDI (2004) A simple mechanistic model seed dispersal, predation and plant establishment: Janzen-Connell and beyond. Journal of Ecology 92: 733-746.

READER RJ (1993) Control of seedling emergence by ground cover and seed predation in relation to seed size for some old-field species. Journal of Ecology 81: 169-175.

RECHENE C, J BAVA \& R MUJICA (2003) Los bosques de Araucaria araucana en Chile y Argentina. Programa de Apoyo Ecológico (TOEB), GTZ, Agencia de Cooperación Alemana, Informe TWFV/40s, Eschborn, Alemania.
SANGUINETTI J (2008) Producción y predación de semillas, efectos de corto y largo plazo sobre el reclutamiento de plántulas. Caso de estudio: Araucaria araucana. Tesis doctoral, Universidad Nacional del Comahue, Bariloche, Argentina. $135 \mathrm{pp}$.

SANGUINETTI J \& T KITZBERGER (2008) Patterns and mechanisms of masting in the large-seeded southern hemisphere conifer Araucaria araucana. Austral Ecology 33: 78-87.

SANGUINETTI J \& T KITZBERGER (2009) Factors controlling seed predation by rodents and nonnative Sus scrofa in Araucaria araucana: potential effects on seedling establishment. Biological Invasions DOI 10.1007/s 10530-009-9474-8.

SCHUPP EW (1990) Annual variation in seedfall, postdispersal predation and recruitment of the neotropical tree. Ecology 71: 504-515.

SHEPHERD JD \& DITGEN RS (2005) Human use and small mammals communities of Araucaria forests in Neuquén, Argentina. Mastozoología Neotropical 12: $217-226$

SHEPHERD JD, RS DITGEN \& J SANGUINETTI (2008) Araucaria araucana and the Austral parakeet: predispersal seed predation on a masting species. Revista Chilena de Historia Natural 81: 395-401.

SILMAN MR, JW TERBORGH \& RA KILTIE (2003) Population regulation of a dominant rain forest tree by a mayor seed predator. Ecology 84: 431-438

SILVERTOWN J (1980) The evolutionary ecology of the mast seeding in trees. Biological Journal of the Linnaean Society 14: 235-250.

SMITH CC, JL HAMRICK \& CL KRAMER (1990) The advantage of mast years for wind pollination. American Naturalist 136: 154-166.

SOKAL RR \& J ROHLF (1994) Biometry: The principles and practice of statistics in biological research. Third Edition, W.H. Freeman and Company Editors, New York. 881 pp.

TILMAN D (1994) Competition and biodiversity in spatially structured habitats. Ecology 75: 2-16.

TURNBULL LA, MJ CRAWLEY \& M REES (2000) Are plant populations seed-limited? A review of seed sowing experiments. Oikos 88: 225-238.

VEBLEN TT (1982) Regeneration patterns in Araucaria araucana forest in Chile. Journal of Biogeography 9: 11-28.

VEBLEN TT (1992) The regeneration dynamics. En: Glenn-Lewin DC, RK Peer \& TT Veblen (eds) Plant succession: Theory and prediction: 152-187. Chapman and Hall, London.

WADA N (1993) Dwarf bamboos affect the regeneration of zoochorous trees by providing habitats to acornfeeding rodents. Oecologia 94: 403-407.

WALLER DM (1979) Models of mast fruiting in trees. Journal of Theoretical Biology 80: 223.

WOLFF JO (1996) Population fluctuations of mast-eating rodents are correlated with production. Journal of Mammalogy 77: 850-856.

Editor Asociado: Julio Gutiérrez.

Recibido el 2 de septiembre de 2008; aceptado el 28 de mayo de 2009 
